\title{
Migration from Microwave to Millimeter-wave for Next Generation Mobile Communication Systems
}

\author{
Felix Opeyemi Olowononi \\ Department of Electrical and Information Engineering, \\ Covenant University, Ota, Nigeria.
}

\author{
Charles Ndujiuba \\ Department of Electrical and Information Engineering, \\ Covenant University, Ota, Nigeria.
}

\begin{abstract}
Communications systems have continued to evolve after ground-breaking research works by various researchers in the 19th and 20th centuries. Mobile wireless communication networks due to their inherent advantages and the availability of ubiquitous broadband internet along with other technological developments in semiconductor science and the convergence of technology have continued to generate an insatiable demand for higher data rates. Since the bandwidth/frequency spectrum is finite and hence limited in availability to telecommunication operators, various techniques have been proffered as solutions to the capacity problem from the first to the fourth generations $(1 \mathrm{G}-4 \mathrm{G})$. However, as the data explosion continues to the big-data age, there is a need to migrate from the utilization of the microwave frequencies to the millimeter-wave frequency to solve the capacity issue. This principle is the core behind the Fifth Generation (5G). This paper is therefore set to present the millimeter-wave technology and its contribution to the expectations for Next Generation Networks (NGN).
\end{abstract}

\section{Keywords}

Fifth Generation, Heterogeneous networks, Microwave, Millimeter wave, Mobile Communications, Next Generation (NGN), Spectrum

\section{INTRODUCTION}

Radio communications involves the transmission, emission and reception of signals (writings, images, sounds or intelligence) through electromagnetic waves at frequencies lower than $3000 \mathrm{GHz}$ and propagated through space without an artificial guide [1]. Modern radio communications or Mobile Communications can be traced to the foundational works of James Clerk Maxwell, Heinrich Rudolph Hertz and Gugliemo Marconi among others. [2, 3].

Mobile communication systems have evolved from cordless systems, paging systems to successive generations of cellular systems. The recent developments in optical communications raised concerns about the future of wireless communication systems. These concerns hinged on the inherent challenges experienced in the use of the wireless or mobile communications systems which include the scarcity of the wireless spectrum, the need for sharing among users, the unreliable nature of the transmission medium due to noise and interference, attenuation of transmitted signal with distance and physical obstructions leading to multipath propagation. However, despite the listed drawbacks of the wireless mobile communication systems, its ubiquitous nature coupled with other factors like the development of high power smart phones and tablets has caused its continuous growth.

Research has shown that as optical communications continue to gain popularity in the backhaul section of communication systems, the last mile access will still be dependent on the mobile communication devices for a while to come. According to the International Telecommunications Union (ITU), the number of mobile cellular subscriptions at the end of 2016 was given as 7.377 billion. This figure corresponds to a $97 \%$ penetration rate and is a far improvement when compared to the 738 million subscriptions in the year 2000 . Global internet penetration rate in the $2000-2015$ timeline has also risen from $6.5 \%$ to $43 \%$. Furthermore, mobile broadband penetration reached $47 \%$ while fixed broadband growing at a slower pace reached $11 \%$ both at the same time [4]. With the increase in mobile subscribers, data consumption has continued to grow in tandem. It is pertinent to note that mobile data traffic grew by 65\% from Q3 2015 to Q3 2016; largely as a result of increased Smartphone consumption hence increased video consumption on mobile devices and social network. In 2015, video and social networking accounted for $50 \%$ and $15 \%$ of mobile data traffic respectively $[5,6]$. Due to developments in Machine-toMachine Communications (M2M) and Internet-of-Things (IoT), there are projections that about 28 billion devices (including consumer electronics) are expected to be connected by 2021 [5].

The above projections imply that call for increased spectrum allocation will continue to rise to cope with the bandwidthhungry age. Despite the campaign against underutilization of available bandwidth and calls for spectrum optimization by the local and international regulation bodies, it is clear that other alternatives must be sought to cope with the trend [6]. One of these solutions is that higher frequency bands in the millimeter wave must be exploited to achieve the desires of Next Generation Networks as research must increase to alleviate for the obvious challenges that will be encountered.

\section{GENERATIONAL APPROACHES TO SPECTRUM/CAPACITY CONSTRAINTS}

Spectrum has been identified as the livewire of telecommunications. The early communication systems like paging and cordless systems were efficient enough at the time because they were used for transmission of voice only and the number of users was also limited. However, as the subscribers began to increase and the desire for additional services grew, research groups in the European, North American and Asian regions of the world sprung up to develop solutions to the challenges encountered.

A major breakthrough was the introduction of the Cellular technology by the Bell laboratories. Contrary to conventional radio telephone networks where a few base stations were used to cover large geographical areas, high capacity is achieved in cellular systems by limiting the coverage area of each base station to a small area known as a cell such that the same radio channels may be used at a distance away. This principle known as the Frequency Reuse System increases the spectral 
efficiency of the system provided the Co-Channel Interference (CCI) can be mitigated [7, 8]. First generation mobile cellular systems like Nordic Mobile Telephony (NMT), Total Access Communication System (TACS) and Advance Mobile Phone System (AMPS) were developed from the idea of the Frequency Reuse Concept. The frequency reuse concept attended to virtually all capacity problems of the first generation since it was based on an analogue technology and utilized circuit switching for voice communication only. Figure 1 below show the evolution of generations of mobile communication systems.

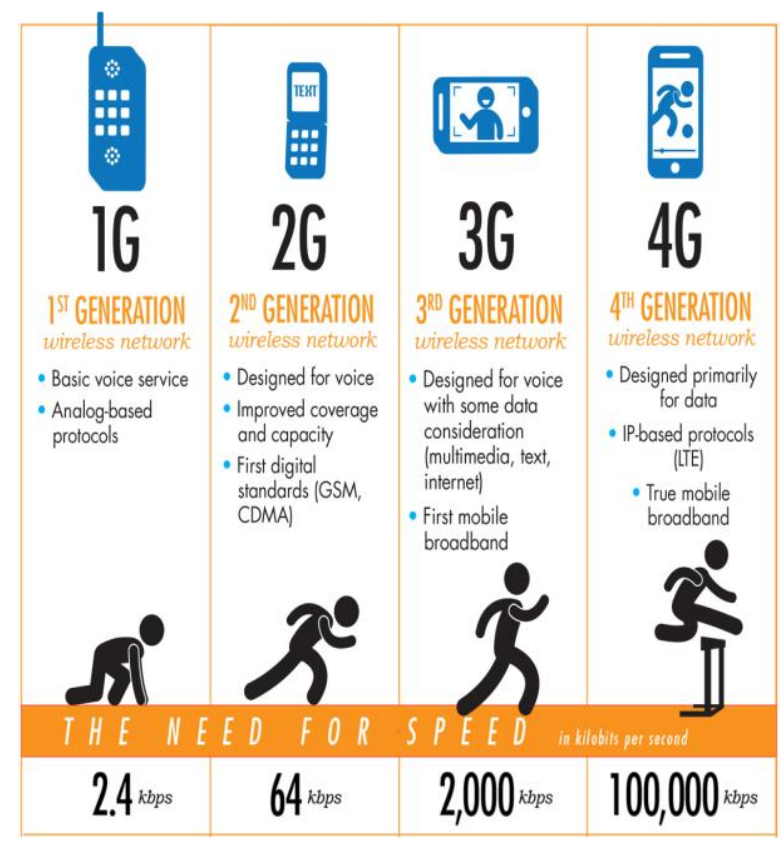

Figure 1: Evolution of Mobile Networks

With time, breakthrough in research towards developing another technology that will offer services like roaming and send short message services resulted in the second generation networks. Enhancements to improve its efficiency and attend to the capacity problem led to the jettisoning of the analog circuit-switched for the digital packet-switched technology, introduction of the Time Division Multiple Access (TDMA) to complement the Frequency Division Multiple Access (FDMA) and the use of digital modulation schemes like GMSK in GSM systems. Other improvements include microcells deployment, frequency borrowing, frequency hopping, cell splitting and cell sectoring [9-11]. Frequency hopping contributes to the resolution of the capacity problem by adding transceivers to existing cells thereby leading to frequency diversity. Cell sectoring however boosts spectral efficiency and Signal-to-Interference Ratio by replacing a single Omni-directional antenna with several directional antennas thus dividing a cell into sectors and in essence increasing the frequency reuse.

Although there were intermediate technologies after the second generation networks, both the GSM and CDMA camps formed their own separate $3 \mathrm{G}$ partnership projects known as 3gpp and 3gpp2 respectively to develop IMT-2000 compliant standards based on the Code Division Multiple Access technology. The $3 \mathrm{~g}$ standard in 3gpp is referred to as Wideband Code Division Multiple Access because it uses a larger $5 \mathrm{MHz}$ bandwidth relative to $1.25 \mathrm{MHz}$ bandwidth used in 3gpp2's cdma2000 system.
The need to meet the continued traffic growth demands led the 3G Partnership Project (3GPP) to begin to develop a new standard for the evolution of GSM/HSPA technology towards a packet-optimized system known as Long Term Evolution (LTE). The goal was thus to produce a new radio access technology with reduced delay with respect to connection establishment and transmission latency, higher user data rates, improved spectral efficiency, increased cell-edge bit-rate, a simplified network architecture, seamless mobility and a reasonable mobile terminal power consumption [12]. One of the ways LTE is able to optimize spectrum allocation is its ability to operate in a wide range of frequency bands and sizes of spectrum allocations in both uplink and downlink. In actual fact, the principle of "Spectrum Refarming" make LTE deployable in spectra currently being occupied by older radio access technologies [13].

Table 1: LTE System Attributes [14]

\begin{tabular}{|c|c|}
\hline Bandwidth & $1.25-20 \mathrm{MHz}$ \\
\hline Duplexing & FDD, TDD, half-duplex FDD \\
\hline Mobility & $350 \mathrm{Km} / \mathrm{h}$ \\
\hline $\begin{array}{l}\text { Multiple } \\
\text { Access }\end{array}$ & $\begin{array}{ll}\text { Downlink } & \text { OFDMA } \\
\text { Uplink } & \text { SC-FDMA }\end{array}$ \\
\hline MIMO & $\begin{array}{l}\text { Downlink: } 2 \times 2,4 \times 2,4 \times 4 \\
\text { Uplink: } \quad 1 \times 2,1 \times 4\end{array}$ \\
\hline $\begin{array}{l}\text { Peak data rate } \\
\text { in } 20 \mathrm{MHz}\end{array}$ & $\begin{array}{ll}\text { Downlink } & 173 \text { and } 326 \mathrm{Mb} / \mathrm{s} \text { for } 2 \times \\
& \begin{array}{l}2 \text { and } 4 \times 4 \text { MIMO } \\
\text { respectively }\end{array} \\
\text { Uplink } & \begin{array}{l}86 \mathrm{Mb} / \mathrm{s} \text { with } 1 \times 2 \\
\text { antenna configuration }\end{array}\end{array}$ \\
\hline Modulation & QPSK, 16-QAM, 64-QAM \\
\hline $\begin{array}{l}\text { Channel } \\
\text { Coding }\end{array}$ & Turbo code \\
\hline
\end{tabular}

The key technologies introduced to make the above features of LTE attainable as summarized in the table above are briefly discussed below. The system is able to support flexible bandwidth due to the employment of advanced multi-carrier air-interface techniques such as Orthogonal Frequency Division Multiple Access (OFDMA) in the uplink and Single Carrier-Frequency Division Multiple Access (SC-FDMA) in the downlink. OFDM subdivides the bandwidth available for signal transmission into a multitude narrowband subcarriers arranged to be mutually orthogonal. The subcarriers when utilized by multiple users guarantees flexibility with the implication that different spectrum bandwidths can be utilized while maintaining the integrity of the equipment design and fractional frequency reuse and interference coordination between cells are facilitated.

The use of multiple antenna technology in LTE systems is another technology that has distinguished it from earlier systems. The three fundamental benefits of Multiple-Input Multiple-Output (MIMO) technology include diversity gain, array gain and spatial multiplexing gain [15]. 
Other technologies employed in the $4 \mathrm{G}$ systems include Hybrid Automatic Repeat Request (ARQ), Adaptive scheduling in both the frequency and spatial domains, Link adaptation of modulation and code-rate and several modes of fast channel state reporting [14].

\section{MICROWAVE APPLICATIONS IN COMMUNICATIONS SYSTEM}

Radio waves and microwaves play an important role in our modern environment. Microwave signals travel by line of sight are not bent by the ionosphere as are lower frequency signals and thus satellite and terrestrial communication links with very high capacities are possible.

Furthermore, various molecular, atomic, and nuclear resonances occur at microwave frequencies, creating a variety of unique applications in virtually all areas of human endeavor. Television signals are transmitted around the globe by satellites using microwaves while airliners are guided from the point of takeoff all the way to landing by microwave and radar navigation systems. Furthermore, Radar systems are used for detecting and locating targets and for air traffic control systems, missile tracking radars, automobile collision avoidance systems, weather prediction, motion detectors, and a wide variety of remote sensing systems. The military serves as a major beneficiary of this technology as they use microwave for surveillance, guidance and control, communication and identification in their tanks, ships and planes.

Microwave communication systems handle a large fraction of the world's international and other long haul telephone, data and television transmissions. Most of the currently developing wireless telecommunications systems, such as direct broadcast satellite (DBS) television) and cellular video (CV) systems rely heavily on microwave technology.

The growth in mobile broadband has a massive impact on the transmission capacity needed to serve the various flavors of radio access network. The three major components for backhaul networks for wireless media are copper, fiber and microwave. Since copper is not scalable enough to meet future demands, fiber will replace copper and wireless media wherever its additional cost and deployment complexity can be justified. However, presently microwave transmission dominates mobile backhaul, where it connects some 60 percent of all macro base stations [16]. Even as the total number of connections grows, the role of the microwave will not be limited to the backhaul network only but also in the last mile access and a complementary role the aggregation part of the network.

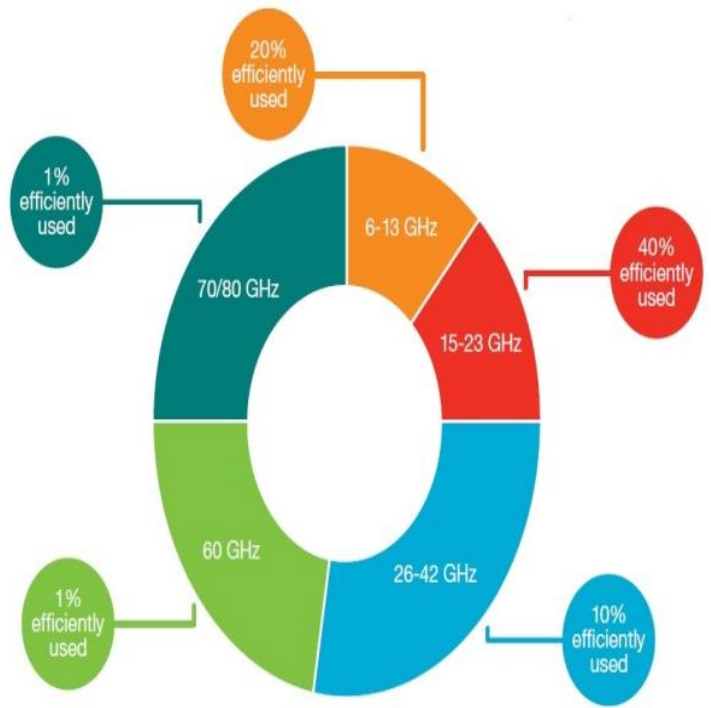

Figure 2: Relative amount of spectrum available per frequency range [Source: Ericsson (2015)]

As shown in figure 2, the traditional bands for microwave communications are around the $0-30 \mathrm{GHz}$ frequency band. The new band and future bands lie in the millimeter-wave frequency band hence the need for the migration from microwave to millimeter-wave frequency to support the heavy data and bandwidth-hungry Next Generation Networks (NGN). It can also be seen that the $60-80 \mathrm{GHz}$ spectrum band which corresponds to the mm-wave frequency range has sparsely been utilized.

\section{MILLIMETER-WAVE FOR NEXT GENERATIONS MOBILE NETWORKS}

Millimeter waves occupy the frequency spectrum between 30 $\mathrm{GHz}$ and $300 \mathrm{GHz}$. This implies that they're located between microwaves and infrared (IR) waves. Their wavelength is in the one millimeter to ten-millimeter range, hence the origin of their names.



Figure 3: Microwave vs Millimeter wave frequency bands for mobile communication

However, in the context of wireless communication, the term generally corresponds to a few bands of spectrum near 38, 60 and $94 \mathrm{GHz}$, and more recently to a band between $70 \mathrm{GHz}$ and $90 \mathrm{GHz}$ that have been allocated for the purpose of wireless communication in the public domain. All of these bands of spectrum correspond to the E-band where there is presently a lot of research interest. The different wave bands in the millimeter wave range are as listed in the table below. 
Table 2. Millimeter-Wave Band Designation

\begin{tabular}{cc}
\hline Designation & Frequency Range $(\mathbf{G H z})$ \\
\hline$Q$-Band & $33-50$ \\
$U$-Band & $40-60$ \\
$V$-Band & $50-75$ \\
$E$-Band & $60-90$ \\
$W$-Band & $75-110$ \\
$D$-Band & $110-170$ \\
$G$-Band & $140-220$ \\
$Y$-Band & $220-325$ \\
\hline
\end{tabular}

Millimeter wave technology, despite its recent application in wireless communication is not a totally new one. Its history can be traced back to the 1890's when J.C. Bose was experimenting with millimeter wave signals at just about the time when his contemporaries like Marconi were inventing radio communications. In the years succeeding Bose's research, millimeter wave technology was confined to the researchers in the university and government laboratories. However, in the 80's, the development of millimeter-wave integrated circuits created opportunities for mass manufacturing of millimeter wave products for commercial applications.

\subsection{Motivations for Millimeter-Wave Wireless Communications}

As earlier highlighted, millimeter wave technology was earlier discovered but its application to mobile communication and networks was limited due to certain constraints. There are however some benefits of communications at the millimeter wave spectrum.

(i) The underutilization of the radio spectrum at the 3- $300 \mathrm{GHz}$ frequency band, the availability of more bandwidth at these frequencies and the growing congestion at the crowded microwave frequency band is a major factor responsible for increased research work at mm-wave frequencies.

(ii) The principle of frequency reuse revolutionized the mobile communication industry by birthing cellular communication systems which significantly tackled the capacity constraint. It is therefore a delight the higher attenuation at mm-wave frequencies that inhibit their distance of travel in free space and other media implies the possibility of frequency reuse at shorter distances hence further increasing capacity.

(iii) The very large magnitude of the frequency at the millimeter wave engenders a very small wavelength thereby implying a corresponding small physical size physical of the antennas needed for transmission. The sizes are so small that the possibility of building complex antenna arrays hence exploiting the principle of MIMO.

(iv) The security concerns in the microwave based technologies are adequately addressed at the mm-wave frequencies as a result of the limited range (short wavelength) and the relatively narrow beam width achievable.

\subsection{Propagation Loss concerns at the Millimeter Wave Spectrum}

The goal of every Mobile Communication system or network is to address the core issues of coverage, capacity and quality in the most cost-effective approach attainable. With respect to capacity and quality, the signal transmitted from the transmitting antenna to the receiving antenna travels along a complex path due to its exposure to a diverse human made and other natural obstacles. These factors tend to attenuate the signal thus affecting its coverage and quality. Propagation models have been used to predict path loss by predicting the average received signal strength at a given distance from the transmitter.

For the macro-cell coverage planning in the microwave frequencies, the Okumura-Hata model is commonly used. The loss between the transmitting and receiving antenna is as expressed in equation (i) below.

$L=$

$A+B \log f-13.82 \log h_{b t s}-$

$a\left(h_{m}\right)\left(44.9-6.55 \log h_{b}\right) \log d$

Where $\boldsymbol{f}$ is the frequency in MHz, $\boldsymbol{h}$ is the BTS antenna height $(\mathrm{m}), \boldsymbol{a}(\boldsymbol{h})$ is a function of the MS antenna height and $\boldsymbol{d}$ is the distance between BS and MS(km). The constants A and B vary with frequencies.

$a\left(h_{m}\right)=\left(1.1 \log f_{c}-0.7\right) h_{m}-\left(1.56 \log f_{c}-0.8\right)$

On the other hand, for micro-cell coverage planning especially in urban environments, the Walfish-Ikegami propagation model is the most commonly used. It is suitable for a frequency range of $800-2000 \mathrm{MHz}$, heights up to $50 \mathrm{~m}$ and distances up to $5 \mathrm{~km}$. The path loss in NLOS conditions is as expressed in equation (iii) below.

$P=32.4+20 \log f+20 \log d+L_{r d s}+L_{m s}$

The atmospheric attenuation of radio waves is largely a function of the frequency of transmission. At the millimeter wave frequency, attenuation is increased in free space and through walls hence the signal is not able to travel for a long distance. This challenge already being faced in the microwave frequencies is however more severe in the millimeter wave.

The range of millimeter waves is adversely affected by the atmosphere. Other factors like rain, fog and moisture in the air are also contributors to the high signal attenuation suffered by millimeter waves. The major effect of this is a reduction in transmission distances. From Figure 4 below, Oxygen absorption is responsible for the high peak in attenuation at $60 \mathrm{GHz}$. Water absorption is also responsible for other peaks.

In the spectrum from $70 \mathrm{GHz}$ to $100 \mathrm{GHz}$, atmospheric attenuation is relatively low (around $0.5 \mathrm{~dB} / \mathrm{km}$ )when compared with other regions A clear atmospheric window can be seen in the spectrum from around $70 \mathrm{GHz}$ to $100 \mathrm{GHz}$. In this area, low atmospheric attenuation around $0.5 \mathrm{~dB} / \mathrm{km}$ occurs, close to that of the popular microwave frequencies, and very favorable for radio transmission. Frequencies above $100 \mathrm{GHz}$ are also unsuitable for radio transmission due to other molecular absorption. 


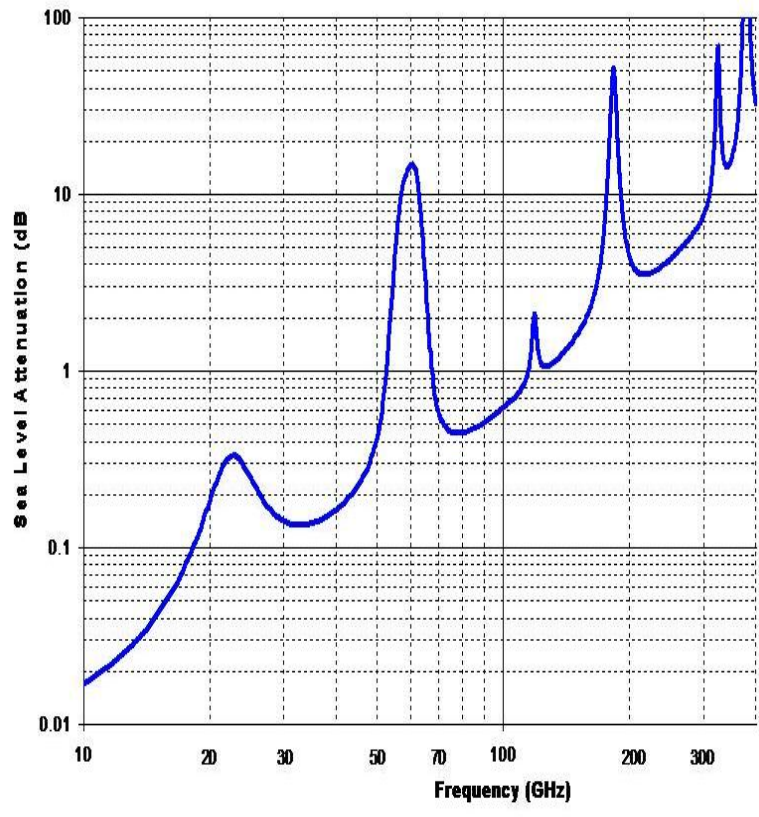

Figure 4: Graph of attenuation in clear weather at the microwave and millimeter frequencies

The higher attenuation at the millimeter wave frequency can be exploited for good in that the same frequency can be reused at shorter distance thereby increasing the number of users that can be accommodated and hence the capacity of the system. This can however be mitigated by designing antennas with good receiver sensitivity, high transmit power and high gain.

\subsection{Underlying Technologies behind Millimeter-wave Utilization}

\subsubsection{Massive Multiple-Input Multiple-Output}

Technology (MIMO) Technology

The concept of the MIMO technology brought a revelation into the antenna design and hence the efficiency of the mobile communication systems. It implies the combinations of multiple transmitters/receivers or antennas at both sides of the digital communication system. Furthermore, the concept of the multiuser MIMO (MU-MIMO) in cellular system which introduces the ability to communicate with several terminals at the same time bring improvements in terms of increase in data rate, reliability as a result of more distinct paths of signal propagation, energy efficiency due to the ability of the base station to focus the emitted energy towards the location of the terminals.

Massive MIMO as the name implies goes beyond the conventional MIMO systems to a new order of systems that use antenna arrays with a few hundred antennas serving many terminals in the same time-frequency resource at the same time. Benefits of massive MIMO include the extensive use of inexpensive low-power components, reduced latency, simplification of the media access control (MAC) layer, and robustness to interference and intentional jamming. The anticipated throughput depends on the propagation environment. The challenge of making many low-cost lowprecision components work effectively together, the need for efficient acquisition scheme for channel state information, resource allocation for newly-joined terminals, the exploitation of extra degrees of freedom provided by an excess of service antennas, reducing internal power consumption to achieve total energy efficiency reductions, and finding new deployment scenarios [17-18] are research issues associated with the use of massive MIMO.

\subsubsection{Spatial Modulation}

One of the techniques that the fifth generation of mobile communication systems will leverage on to meet the everincreasing demand for high-bandwidth data capacity is the spatial modulation. Spatial Modulation proposed by [19] is an extension of conventional modulation where the constellation with three dimensions is constructed.

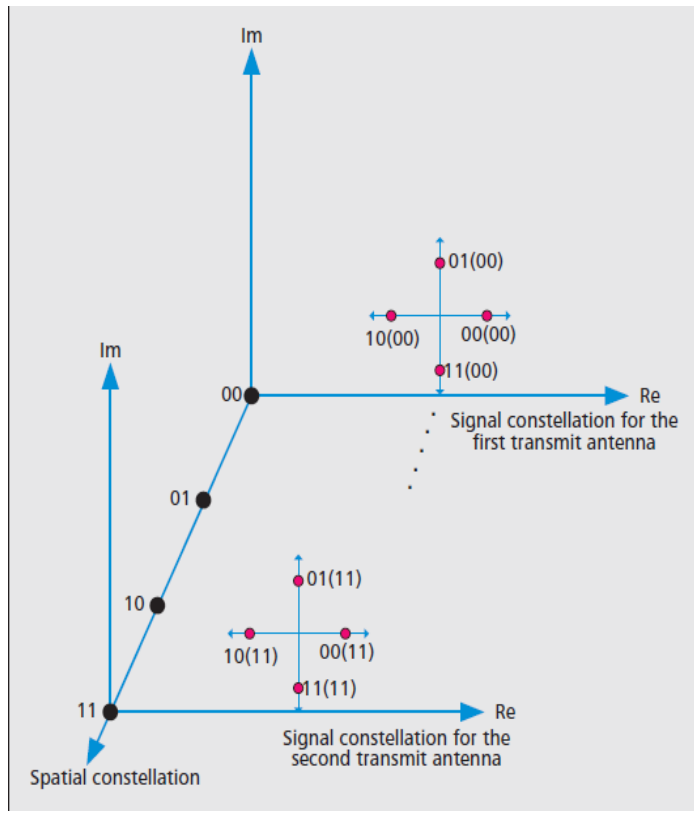

Figure 5: 3-Dimensional Constellation Diagram for Spatial Modulation

Although Multiple-input Multiple-output (MIMO) wireless systems provide a good path for increasing the volume of data transferable to single- or multiple-user environment, they have the tendency of suffering from Signal-to-Noise-Ratio (SNR) degradation at higher Quadrature-Amplitude-Modulation (QAM) orders. Other challenges associated with the VBLAST space time coding scheme of MIMO technology is the high Inter-Channel Interference at the receiver thereby necessitating complex receiver algorithm (which in turns affects the level of power consumption by the mobile system) and the need to have the number of receivers equal or greater than the transmit antennas.

Research by [20] concluded that the Spatial ModulatedMIMO technique performed better in SNR and bit-error-rate (BER) tests over other commonly used massive-MIMO algorithms. The key is that SM-MIMO requires a lower QAM alphabet order compared to massive MIMO at the same spectral efficiency, which is a function of the increased spatial streams per user. These factors lead to higher spatial interference with traditional massive-MIMO systems.

\subsubsection{Heterogeneous Networks}

The traditional response to requests for increased data rates and improved system capacity in HSPA or LTE is to upgrade the radio access, seek for additional spectrum, provide more antennas and advanced processing techniques within and between nodes. 
The concept of the Heterogeneous Network or HetNet has arisen out of the need for cellular telecommunications operators to be able to operate networks consisting of a variety of radio access technologies, formats of cells and many other aspects, and combining them to operate in a seamless fashion.

Another method is to complement the macro networks with low-power nodes such as micro and pico base stations thereby creating a heterogeneous network. The enhanced performance of this arrangement is guaranteed by the fact that low power nodes serve a significant number of hotspots and coverage holes. The challenge however with deploying low power nodes is the need for proximity to the points of traffic generation and the need for numerous units due to their reduced range of coverage. These challenges are however surmountable by effective design and integration of the low power nodes.

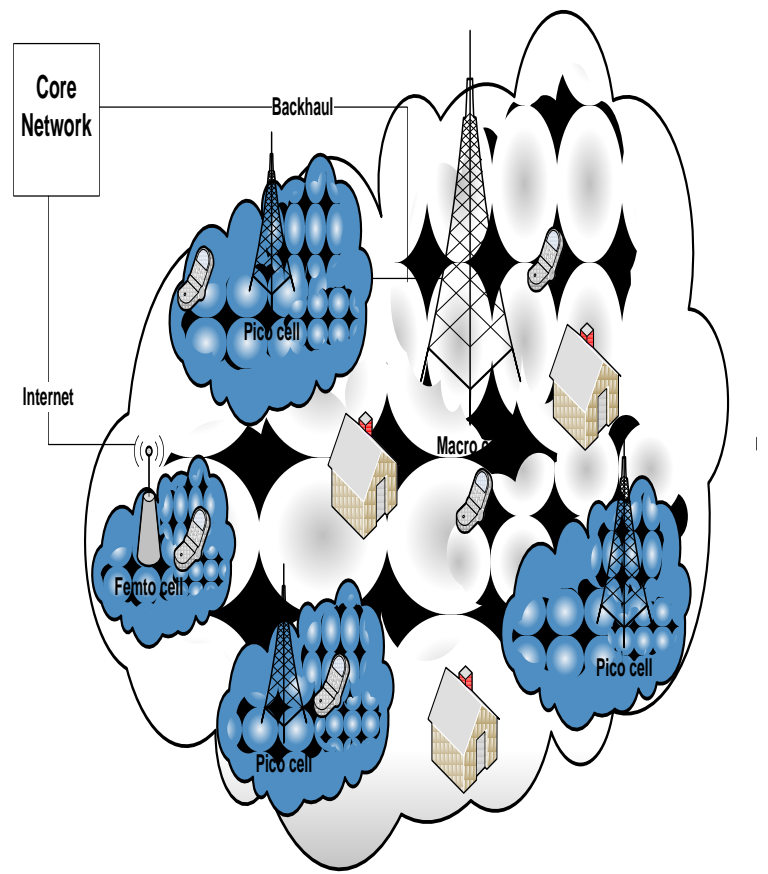

Figure 6: A Heterogeneous Network (HetNet)

\subsubsection{Semiconductor Technology (Gaas)}

Semiconductor technology is a very important catalyst of advancements in computing and communications. Their role in the developments of Integrated Circuits and other miniaturization techniques has fast reduced the size of our access devices and the cost also. One of the reasons the utilization of the millimeter wave was first adjudged impossible was the lack of devices to generate, transmit and receive it. The silicon based devices in microwave communications has caused revolutions in the transmitter, amplifier, receiver, waveguide and repeater technologies.

However, recent developments in semiconductor engineering are encouraging the use of millimeter wave in mobile communications. Although Silicon is the dominant semiconductor for electronics applications today, othe semiconductors like Gallium Arsenide (GaAs), Indium Phosphide (InP), Gallium Nitride (GaN) and Silicon Germanium (SiGe) exhibit superior electron velocity, mobility characteristics hence higher efficiency and lower parasitic resistance at millimeter waves.

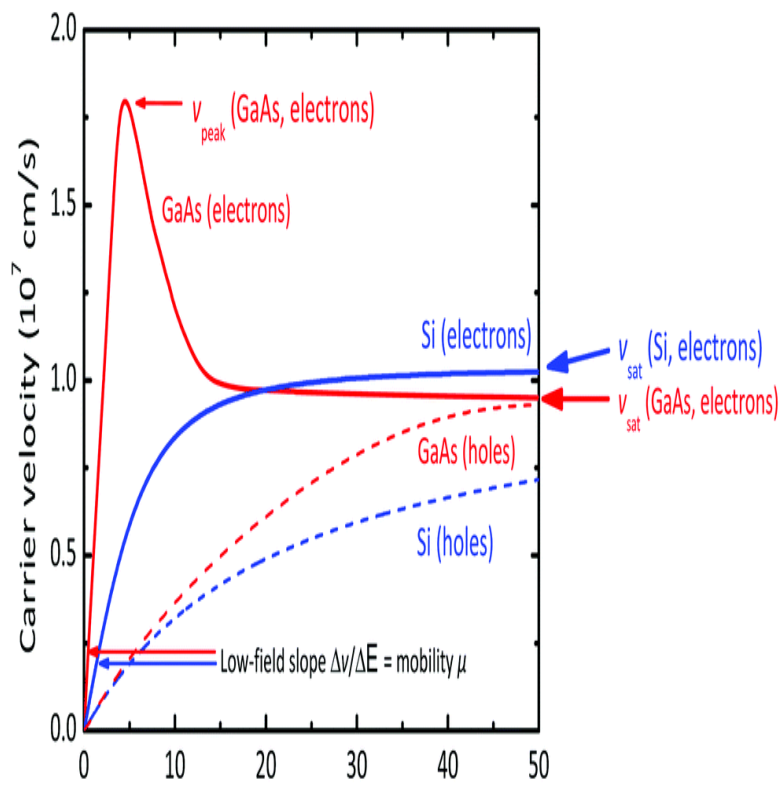

Electric field $(\mathrm{K} / \mathrm{/m})$

Figure 7: Electron Velocity for several semiconductors

\section{CONCLUSION}

The continuous developments in computing and communications technologies will continue to extend the big data revolution. Some of these developments include Machine to Machine communications, Internet of Things, autonomous cars, remote controlled industrial robots, Tele-health systems and smart city infrastructure. An implication of this development is demand for more capacity and hence demand for more wireless spectrum. The scarcity of spectrum is among the factors pushing for a migration from the microwave to the millimeter frequency spectrum where bandwidth is more available despite its associated challenges. The new generation of mobile communication systems that will leverage on this technology have been termed the fifth generation with an expectation to deliver 1Gbps downlink initially and a latency of one millisecond among others. The underlying technologies that will be employed to make this migration possible have been discussed in this paper. The potential of the application of millimeter wave in mobile communication systems is enormous and when well employed, it might not be out of place to say that there will be no need for a $6 \mathrm{G}$.

\section{REFERENCES}

[1] Bangaly Fode Traore, "Radio Regulations", Paper presented at ITU-ATU Regional Radio communication Seminar for Africa, Niamey, Niger, 20 - 24 April, 2015. Available Online: https://www.itu.int/en/ITUR/seminars/rrs/2015-Africa/SeminarTerrestrial/RRS15Africa-Radio\%20Regulation.pdf

[2] Harris Corporation, RF Communications Division, "Radio Communications in the Digital Age", Volume One: HF Technology, Edition 2, (C) Harris Corporation 2005. Available Online: http://rf.harris.com/media/Radio\%20Comms\%20in\%20t he\%20Digital\%20Age\%20-\%201_tcm26-12947.pdf

[3] Richard Frenkiel, 'A Brief History of Mobile Communications', Available Online: 
http://www.winlab.rutgers.edu/ narayan/Course/Wireles s_Revolution/vts\%20article.pdf

[4] ICT Facts and Figures, The World in 2015, A publication of ICT Data and Statistics Division, Telecommunication Development Bureau OInternational Telecommunication Union, May 2015. Available Online: http://www.itu.int/en/ITU-

D/Statistics/Documents/facts/ICTFactsFigures2015.pdf

[5] Ericsson Mobility Report: On the Pulse of the Networked Society, November 2015 @ Ericsson AB 2015, Available Online:

http://www.ericsson.com/res/docs/2015/mobility-

report/ericsson-mobility-report-nov-2015.pdf

[6] A Cisco Systems White Paper, "Cisco Virtual Networking Index: Global Mobile Data Traffic Forecast Update, 2015 - 2020" Available Online: http://www.cisco.com/c/en/us/solutions/collateral/service -provider/visual-networking-index-vni/mobile-whitepaper-c11-520862.pdf

[7] Ranja Mishra et al, "Analysis of Co-channel Interference under various Radio Propagation Environment", International Journal of Engineering Research and Applications (IJERA), Vol 3, Issue 2, March - April 2013

[8] Engstrom Stefan, Johansson Thomas and Kronestedt, "Multiple Reuse Patterns for Frequency Planning in GSM Networks"e, IEEE 1998

[9] Nura Muhammad Shehu, "Coverage and Capacity Improvement in GSM Coverage", International Journal of Novel Approach in Electrical and Mechanical Engineering, Vol. 2, Issue 3, December 2015

[10] Sohrab Alam et al, "Capacity Improvement by Cell Splitting Technique in CDMA System over Telecommunication Network", International Refereed Journal of Engineering and Science (IRJES), Volume 2, Issue 7 (July2013), PP.01-08

[11] Ohaneme C.O, Onoh G.N, Ifeagwu E.N, "Improving channel capacity of a Cellular system using Cell Splitting", International Journal of Scientific and Engineering Research, Volume 3, Issue 5, May 2012
[12] Nadim K.M, Zurina M.H, Mohamed Othman and Shamala Subramaniam, "On Multi-cell Packet Scheduling of LTE-A Cellular Networks: A Survey of Concepts Related Challenges and Solutions", Journal of Applied Sciences, 14: 2422-2438. Available Online: http://scialert.net/abstract/?doi=jas.2014.2422.2438

[13] Adu O.I, Idachaba F.E, Alatishe A.A, "Refarming $1800 \mathrm{MHz}$ GSM Spectrum to LTE: The Effects on Coverage Based on Pathloss Estimation", Proceedings of the World Congress on Engineering 2014, Volume 1, WCE 2014, July 2 - 4, 2014, London U.K

[14] Farooq Khan, "LTE for 4G Mobile Broadband: Air Interface Technologies and Performance (C) Cambridge University Press 2009

[15] [15] Ibikunle Frank, Felix Olowononi, "Application of MIMO Technology to systems Beyond 3G", International Journal of Informatics and Communications Technology (IJ-ICT), Vol 1, No 2 (2012) ISSN: 2252-8776 () 2012 IAES Institute of Advanced Engineering and Science

[16] An Ericsson Microwave report, "Microwave Towards 2020: Delivering High-Capacity and Cost-Efficient Backhaul for Broadband Networks Today and in the Future" (C) Ericsson 2015

[17] "Large-scale Multiuser SM-MIMO Versus Massive MIMO," 2014 Information Theory and Applications Workshop (ITA), Feb. 2014, p. 1.

[18] G.J. Foschini, M.J. Gans, "On limits of wireless communications in a fading environment when using multiple antennas," Wireless Personal Commun., vol. 6, pp. 311-335, 1998.

[19] R.Mesleh, H. Haas, C.W. Ahn, and S. Yun , "Spatial Modulation- A New Low Complexity Spectral Efficiency Enhancing Techinique," Proceedings of CHINACOM, Beijing, China, pp.1-5, 2006.

[20] G.D. Golden, G.J. Foschini, R.A. Valenzuela, and P.W. Wolniansky, "Detection algorithm and initial laboratory results using V-BLAST space-time communication architecture," Electron Letter, vol.35, pp.14-16, 1999. 